\title{
CNS-specific immunity at the choroid plexus shifts toward destructive Th2 inflammation in brain aging
}

\author{
Kuti Baruch, ${ }^{a, 1}$, Noga Ron-Harel ${ }^{b, 1}$, Hilah Gal ${ }^{c, 1}$, Aleksandra Deczkowskaa, Eric Shifrutc, Wilfred Ndifon, \\ Nataly Mirlas-Neisberg ${ }^{a}$, Michal Cardon ${ }^{a}$, llan Vaknin ${ }^{a}$, Liora Cahalona, Tamara Berkutzki ${ }^{a}$, Mark P. Mattson ${ }^{d}$, \\ Fernando Gomez-Pinilla ${ }^{\mathrm{e}, \mathrm{f}}$, Nir Friedman ${ }^{\mathrm{c}, 2}$, and Michal Schwartz ${ }^{\mathrm{a}, 2,3}$
}

Departments of a Neurobiology and 'Immunology, Weizmann Institute of Science, Rehovot 76100, Israel; bepartment of Cell Biology, Harvard Medical
School, Boston, MA 02115; ${ }^{\mathrm{d}}$ Laboratory of Neurosciences, National Institute on Aging Intramural Research Program, National Institutes of Health,
Baltimore, MD 21224; and Departments of Integrative Biology and Physiology and ${ }^{\mathrm{f}}$ Neurosurgery, University of California, Los Angeles, CA 90095

Edited* by Michael Sela, Weizmann Institute of Science, Rehovot, Israel, and approved November 13, 2012 (received for review July 4, 2012)

\begin{abstract}
The adaptive arm of the immune system has been suggested as an important factor in brain function. However, given the fact that interactions of neurons or glial cells with $\mathrm{T}$ lymphocytes rarely occur within the healthy CNS parenchyma, the underlying mechanism is still a mystery. Here we found that at the interface between the brain and blood circulation, the epithelial layers of the choroid plexus (CP) are constitutively populated with $\mathrm{CD}^{+}$effector memory cells with a T-cell receptor repertoire specific to CNS antigens. With age, whereas CNS specificity in this compartment was largely maintained, the cytokine balance shifted in favor of the T helper type 2 (Th2) response; the Th2-derived cytokine IL-4 was elevated in the CP of old mice, relative to IFN- $\gamma$, which decreased. We found this local cytokine shift to critically affect the $\mathrm{CP}$ epithelium, triggering it to produce the chemokine CCL11 shown to be associated with cognitive dysfunction. Partial restoration of cognitive ability in aged mice, by lymphopenia-induced homeostasis-driven proliferation of memory T cells, was correlated with restoration of the IL-4:IFN- $\gamma$ ratio at the $\mathrm{CP}$ and modulated the expression of plasticity-related genes at the hippocampus. Our data indicate that the cytokine milieu at the CP epithelium is affected by peripheral immunosenescence, with detrimental consequences to the aged brain. Amenable to immunomodulation, this interface is a unique target for arresting age-related cognitive decline.
\end{abstract}

blood-cerebrospinal fluid barrier | brain senescence | neuroinflammation

irculating immune cells have been repeatedly shown to be essential for central nervous system (CNS) maintenance (1-3). Specifically, T cells that recognize CNS antigens contribute to the functional integrity of the CNS under both normal and pathological conditions $(2,4-6)$, supporting hippocampus-dependent learning and memory, adult neurogenesis, and neurotrophic factor production (2).

Under physiological conditions, $\mathrm{T}$ cells are rarely found in the brain parenchyma and are mainly observed at the borders of the CNS: the choroid plexus (CP) of the brain's ventricles, forming the blood-cerebrospinal fluid barrier (BCSFB), the meningeal spaces, and the cerebrospinal fluid (CSF) (7). T cells were shown to accumulate in these compartments in response to signals from the CNS, specifically in the meninges after performance of cognitive tasks (8) and in the $\mathrm{CP}$ after exposure to mental stress (9). In the meningeal spaces, these cells were further characterized as producing the cytokine interleukin 4 (IL-4), known for its beneficial role in CNS maintenance and neuroprotection $(8,10-13)$. However, the questions of why, where, and how T-cell specificity is needed for brain plasticity remained mysterious.

The CP is strategically positioned at the lining between the CNS and the immune system and, in addition to its classically known role in generating the CSF, can enable bidirectional communication between the CNS parenchyma and blood circulation (14). Accordingly, we envisioned that $\mathrm{T}$ cells that reside in this compartment interact with their relevant antigen-presenting cells (APCs) and respond, upon stimulation, by secreting cytokines that modulate the properties of the $\mathrm{CP}$ epithelium, thereby affecting the CNS parenchyma from afar.
In the present study, we show that the CP in young and aged animals retains $\mathrm{CD} 4^{+}$effector memory cells with a T-cell receptor (TCR) repertoire specific to CNS antigens. With aging, the CP manifested signs of $\mathrm{T}$ helper type 2 (Th2) inflammation, linking its functional plasticity to age-related cognitive decline. Using an immunological manipulation that induced homeostasis-driven proliferation and partially restored cognitive ability, we demonstrate that peripheral rejuvenation of the immune system can immunomodulate the $\mathrm{CP}$, shifting its cytokine balance and modifying hippocampal plasticity.

\section{Results}

The Choroid Plexus Is Populated by Effector Memory $\mathrm{CD}^{+}{ }^{+}$Cells. Using flow cytometry, we found that, similar to the lymph nodes (LNs), the majority $(62 \pm 2.76 \%)$ of $\mathrm{T}$ cells found in CPs were $\mathrm{CD}^{+}$cells (Fig. $1 A$ ). Because $\mathrm{CD}^{+} \mathrm{T}$ cells (rather than $\mathrm{CD}^{+}$ cells) were previously implicated in supporting CNS plasticity $(1$, $2,8,12$ ), we further characterized this subpopulation. We focused our interest on memory $\mathrm{T}$ cells, commonly divided into two subsets (Fig. $1 B$ ): CD44 ${ }^{\text {high }} / \mathrm{CD} 62 \mathrm{~L}^{\text {high }}$ are central memory T cells $\left(\mathrm{T}_{\mathrm{CM}}\right)$, whereas $\mathrm{CD} 44^{\text {high }} / \mathrm{CD} 2 \mathrm{~L}^{-/ \text {low }}$ are effector memory $\mathrm{T}$ cells $\left(\mathrm{T}_{\mathrm{EM}}\right)$ that can be locally activated to an immediate effector function upon encountering their cognate antigen (15). In the CP, unlike the blood and LNs, the vast majority of memory $\mathrm{T}$ cells expressed $\mathrm{T}_{\mathrm{EM}}$ markers (Fig. $1 C$ ). In aged mice (22-mo-old), we observed an increase relative to young mice (3-mo-old) in the frequency of $\mathrm{T}_{\mathrm{EM}}$ cells in both the blood circulation and the LNs, whereas their levels in the $\mathrm{CP}$ were unchanged (Fig. $1 C$ and $D$ ). Thus, unlike the CSF, which is dominated by $\mathrm{CD} 4^{+} \mathrm{T}_{\mathrm{CM}}$ cells $(16$, 17), the $\mathrm{CP}$ specifically retains $\mathrm{CD} 4^{+} \mathrm{T}_{\mathrm{EM}}$ cells throughout life. $\mathrm{T}$ cells in the CP were colocalized with the epithelium (Fig. $1 E$ ) and adjacent to MHCII-expressing APCs (Fig. $1 F$ ), indicating their potential functional interactions there. We therefore assumed that the $\mathrm{T}_{\mathrm{EM}}$ cells that reside in the $\mathrm{CP}$ are activated by APCs that present their cognate antigens, and are likely to be specific for brain antigens. Thus, we next focused on identifying the specificity of the $\mathrm{T}$ cells that reside in the $\mathrm{CP}$ and whether they undergo changes with age that could, to some extent, explain brain senescence.

The Choroid Plexus CD4 ${ }^{+}$TCR Repertoire Is Enriched with CNS-Specific Clones. Sequencing of a TCR by itself cannot identify its antigenic specificity. Therefore, we developed a unique approach that allowed us to identify clonotypic enrichment of CNS-specific T cells. Mice were immunized with spinal cord homogenate $(\mathrm{SCH})$

Author contributions: K.B., N.R.-H., H.G., W.N., N.F., and M.S. designed research; K.B., N.R.-H., H.G., A.D., E.S., W.N., N.M.-N., M.C., I.V., L.C., and T.B. performed research; M.P.M. and F.G.-P. contributed new reagents/analytic tools; K.B., N.R.-H., H.G., A.D., E.S., W.N., and N.M.-N analyzed data; and K.B. and M.S. wrote the paper.

The authors declare no conflict of interest.

*This Direct Submission article had a prearranged editor.

${ }^{1}$ K.B., N.R.-H., and H.G. contributed equally to this work.

${ }^{2}$ N.F. and M.S. contributed equally to this work.

${ }^{3}$ To whom correspondence should be addressed. E-mail: michal.schwartz@weizmann.ac.il.

This article contains supporting information online at www.pnas.org/lookup/suppl/doi:10. 1073/pnas.1211270110/-/DCSupplemental. 
Fig. 1. The Choroid plexus is populated by effector memory $\mathrm{CD}^{+} \mathrm{T}$ cells. (A) Flow cytometry plots of $\mathrm{CD}^{+} / \mathrm{CD}^{+}$T-cell frequencies (mean; \%) in the inguinal LNs and the CPs, pregated by $\mathrm{CD}_{4} 5^{+}$and $\mathrm{TCR}^{+}$( $n=5$ per group). ( $B$ ) Flow cytometry gating strategy for inguinal LN, blood, and CP tissues. Cells were stained for markers of effector $\left(\mathrm{CD} 45^{+}, \mathrm{TCR} \beta^{+}\right.$, $\mathrm{CD}^{+}, \mathrm{CD} 44^{\text {high }}$, and $\left.\mathrm{CD}^{+} 2 \mathrm{~L}^{-}\right)$and central $\left(\mathrm{CD} 45^{+}\right.$, $\mathrm{TCR} \beta^{+}, \mathrm{CD}^{+}, \mathrm{CD} 44^{\text {high }}$, and $\mathrm{CD} 2 \mathrm{~L}^{+}$) memory $\mathrm{CD} 4^{+}$ $T$ cells. FCS, forward scatter; SSC, side scatter. (C) Representative plots for young (3-mo-old) and aged (22-mo-old) mice effector and central memory $\mathrm{CD}^{+}$ T-cell frequencies in inguinal LN, blood, and CP tissues. $(D)$ Quantitative analysis of the data shown in C. Data shown are representative of three independent experiments. Bar graphs throughout the figure show mean \pm SE of each group $(n=4-5$ per group; ${ }^{*} P<0.05 ; * * * P<0.001$; Student's $t$ test). $\mathrm{BL}$,

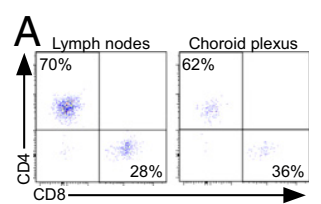

C
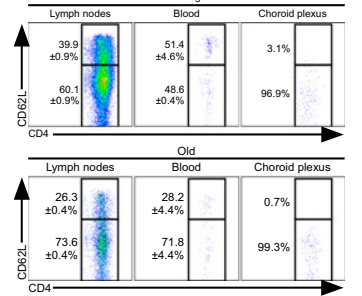

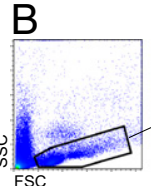

D
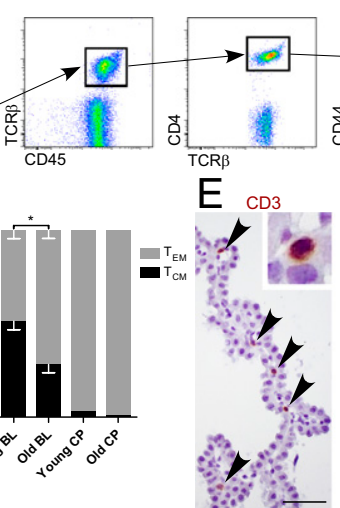

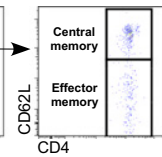

F

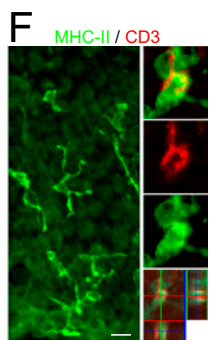

blood. (E) Localization of T cells (stained for CD3; arrowheads) in the CP of naïve animals. (Scale bar, $50 \mu \mathrm{m}$.) ( $F$ ) Confocal images of CP tissues from naïve animals showing the distribution of APCs (stained for MHCII; green) and their colocalization with T cells (stained for CD3; red) using orthogonal projections of confocal z stacks. (Scale bar, $25 \mu \mathrm{m}$.)

containing a wide array of CNS proteins, in order to enrich the spleen with CNS-specific T cells. RNA isolated from splenic $\mathrm{CD}^{+} \mathrm{T}$ cells and CP-resident $\mathrm{T}$ cells was analyzed using TCRseq, a high-throughput sequencing procedure of the TCR $\beta$ CDR3 region (18-20). Using bioinformatics tools developed inhouse (see SI Materials and Methods for a detailed description), full characterization of each CDR3 region was achieved from individual sequencing reads, identifying variable $(\mathrm{V} \beta)$, diversity $(\mathrm{D} \beta)$, and joining $(\mathrm{J} \beta)$ gene segments use. Data obtained were further analyzed by a specially designed analysis pipeline enabling extraction of reliable quantitative information on TCR $\beta$ repertoire composition, providing a list of annotated TCR $\beta$ sequences (nucleotide and amino acid sequences), and their relative abundance, for each sample.

Once we established the TCR $\beta$ repertoire of the spleens of animals immunized with CNS antigens, we compared it to the repertoire of $\mathrm{T}$ cells from the $\mathrm{CP}$ of nonimmunized animals. We observed a high level of similarity in V $\beta$ use between the TCR $\beta$ repertoire found in $\mathrm{CP}$ of naïve animals and that found in the spleens of animals immunized with CNS antigens (SCH) (Fig. S1 $A$ and $B$ ). In contrast, the naïve $\mathrm{CP} \operatorname{TCR} \beta$ repertoire showed much lower correlation with the repertoire found in spleens of naïve or ovalbumin (OVA)-immunized mice. $\mathrm{J} \beta$ use analysis gave similar results (Fig. S1 $C$ and $D$ ).

Next, we applied average-linkage clustering analysis to the different groups, based on the group average relative frequencies of all $\mathrm{V} \beta$ and $\mathrm{J} \beta$ segments (36 parameters overall). The naïve CP formed a distinct linkage cluster with the $\mathrm{SCH}$-immunized spleen, whereas the OVA-immunized spleen clustered with the naïve spleen (Fig. 2A). The repertoire of $\mathrm{T}$ cells residing in the naïve $\mathrm{CP}$ showed a high level of similarity in $\mathrm{V} \beta$ and $\mathrm{J} \beta$ segment use to spleens of SCH-immunized mice, yet not with naive or OVAimmunized spleens. Finally, we examined the naïve $\mathrm{CP}$ repertoire in terms of specific clones. From our TCR-seq data, we compiled a library of 2,752 TCR $\beta$ amino acid sequences that were significantly up-regulated in the SCH-immunized spleens compared with spleens of nonimmunized animals. These clones showed only a small overlapping with OVA-immunized spleens (Fig. S2). Examining the most frequent clones in the naïve $\mathrm{CP}$ revealed that these clones were found in similar frequencies in spleens of CNSimmunized mice but not in naïve spleens (Fig. $2 B$ ). Importantly, in some cases, we observe different nucleotide sequences encoding the same amino acid CDR3 sequence (Fig. $2 B$ ), suggesting selection based on specificity.

We also determined how many of the most highly expressed clones from each analyzed tissue were found in the CNS-specific TCR $\beta$ library (Fig. 2C). The spleens of aged mice were found to be enriched with CNS-specific clones compared with naïve spleens of young mice (Fig. $2 C$ ), possibly reflecting the lifelong exposure to autoantigens believed to result in accumulation of autoreactive clones with age (21). T cells from $\mathrm{CP}$ of aged mice maintained clonotypic specificity to CNS antigens, though the abundance of CNS-specific clones was somewhat lower relative to young CP (Fig. $2 C$ and $D$ ). Following immunization with $\mathrm{SCH}$, the abundance of CNS-specific clones was elevated in the $\mathrm{CP}$ compared with the nonimmunized CP (Fig. 2D), suggesting that the accumulation of CNS-specific T cells at the CP is subject to changes and reflects peripheral immunity.

Th2-Mediated Inflammation of the CP Epithelium During the Aging Process. Based on the above results, we hypothesized that aging of the CP might be associated with changes in T-cell effector phenotype bias, an established phenomenon outside the CNS during aging $(22,23)$. To examine this, we measured mRNA levels of the cytokines ifn- $\gamma$ and $i l-4$ in this compartment, representing the Th1 and $\mathrm{Th} 2$ effector phenotypes, respectively. We found preferential elevation of $i l-4$ expression and a decline in ifn- $\gamma$ expression with aging (Fig. $3 A$ ).

IL-4-producing cells were recently identified at the meningeal spaces of the brain and shown to support cognitive function (8). Outside the CNS, however, IL-4 was shown to induce expression of CCL11 (24), a chemokine associated with age-related cognitive decline, and is elevated in the CSF and plasma of aged mice and humans (25). This apparent contradiction between the beneficial roles of IL-4 in cognitive performance and its known potential to induce CCL11 expression outside the CNS led us to consider a link between the two effects in the aged brain; namely, we envisioned that the age-related CCL11 found in the CSF during aging (25) may be a product of the CP epithelium, resulting from overwhelming levels of IL-4 that develop in this compartment with aging.

We therefore examined mRNA and protein levels of CCL11 in the aged $\mathrm{CP}$, and found them to be elevated (Fig. $3 B$ and $C$ ) relative to young animals. CCL11 immunoreactivity was barely detected in young animals, whereas it was highly abundant in aged CP tissue (Fig. 3D). To understand how the local cytokine balance controls CCL11 induction, we examined its expression by the CP in response to IL-4 in vitro. ccl11 mRNA expression levels by young CP cells were significantly up-regulated in a direct relationship to IL-4 concentrations (Fig. $3 E$ ). Moreover, whereas treatment of young CP epithelial cells with IFN- $\gamma$ alone had no effect on $c c l 11$ expression, addition of IFN- $\gamma$ together with IL-4 reversed the effect of IL-4 on $c c l 11$ production (Fig. $3 F$ ). Intracellular CCL11 immunostaining confirmed that the CP epithelium produced CCL11 in response to IL-4, and that co-incubation with IFN- $\gamma$ reversed this induction (Fig. $3 G$ ). These results confirmed that although young $\mathrm{CP}$ epithelial cells hardly produce CCL11, their exposure to IL-4 up-regulates CCL11 production and IFN- $\gamma$ can mitigate this effect. We therefore tested whether CCL11 production by aged CP is amenable to down-regulation by IFN- $\gamma$. We cultured $\mathrm{CP}$ epithelial cells of young and old mice and monitored their ccll1 expression in the 

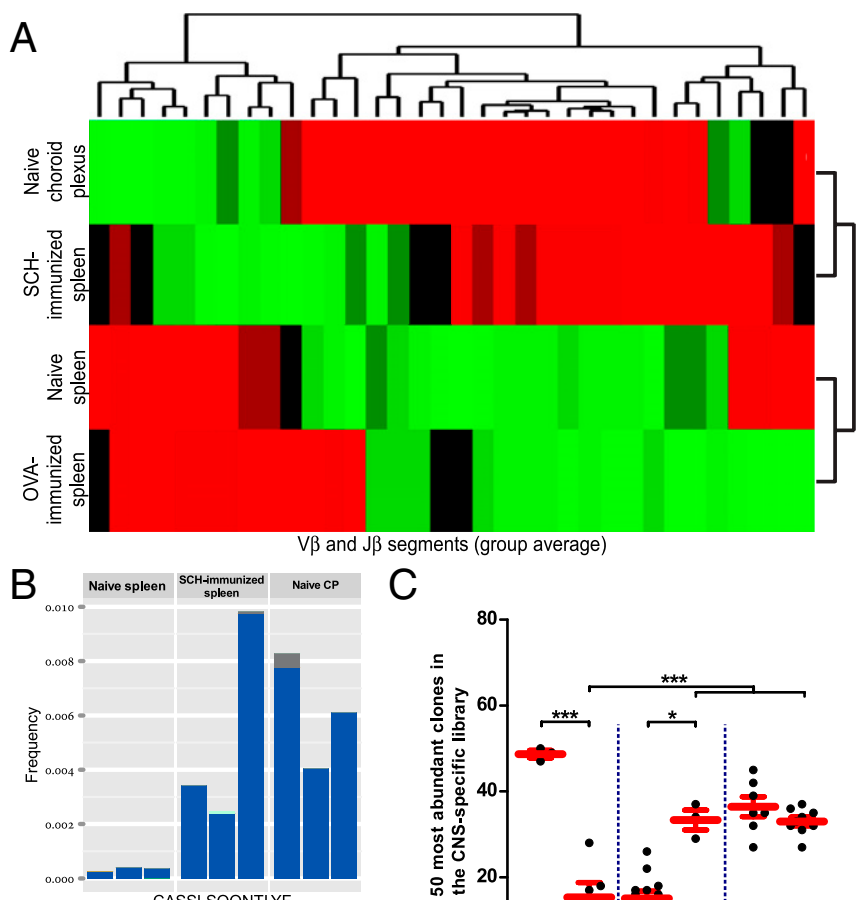

CASSLSQQNTLYF

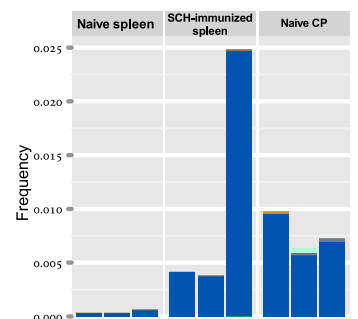

CASSLSGSNYAEQFF

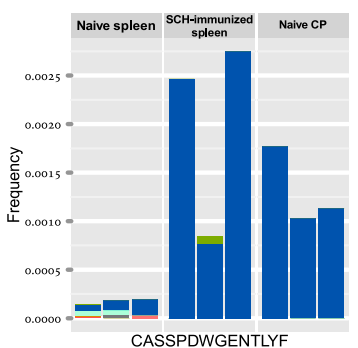

Fig. 2. The choroid plexus CD4 ${ }^{+}$TCR repertoire is enriched with CNS-specific clones. (A) Group hierarchical clustering based on the average $\mathrm{V} \beta$ and $J \beta$ use described in Fig. S1. (B) Examples of TCR $\beta$ clones found to be expanded in the spleen of $\mathrm{SCH}$-immunized mice and in the naïve $\mathrm{CP}$ ( $n=3$ mice per group). Colors indicate different nucleotide sequences that encode the same amino acid CDR3 sequence. (C) A library of CNS-specific clones was compiled from measured TCR $\beta$ amino acid sequences from all mice in the spleen $\mathrm{SCH}$-immunized group. The library contains clones that were significantly expanded compared with the group of naïve mouse spleen. The 50 most abundant clones from each tissue were examined for their presence in this library $(n=$ 3-12 per group; $* P<0.05 ; * * * P<0.001$; one-way ANOVA, Newman-Keuls post hoc). (D) Quantitative comparison between the fractions of clones present in the CNS-specific library using different cutoffs for the most abundant clones of each tissue ( $P<0.05$; two-way ANOVA).

presence or absence of IFN- $\gamma$. In aged CP cultures the basal level of $c c l 11$ was higher than those in young CP (Fig. $3 H$ ), and the addition of IFN- $\gamma$ decreased it to the basal level of young CP culture.

To verify this IL-4-IFN- $\gamma$ interplay at the CP in vivo, we also examined this compartment in IFN- $\gamma$ receptor knockout animals (IFN- $\gamma \mathrm{R}-\mathrm{KO}$ ). We found that the complete absence of
IFN- $\gamma \mathrm{R}$ in the knockout animals resulted in significantly higher ccl11 expression by the $\mathrm{CP}$ (Fig. 3I), further demonstrating the role of IFN- $\gamma$ in balancing IL-4 response in this compartment. Thus, it appears that IL-4-induced CCL11 expression could be balanced by IFN- $\gamma$ in vivo, further suggesting that this regulation might be applicable to the aged CP in vivo.

When applied on astrocytes (8) or microglia (13), IL-4 induces brain-derived neurotropic factor (BDNF), a key molecule in mediating CNS plasticity, learning, and memory. We examined whether IL-4 would have a similar effect on the CP. We found that $b d n f$ was up-regulated in the CP cultures in response to IL-4, up to a certain concentration threshold, beyond which, upon further increase of IL-4 concentration, $b d n f$ levels were reduced (Fig. $3 J$ ). Immunostaining the CP cultures showed the localization of BDNF to the epithelial cells (Fig. $3 K$ ). Thus, whereas high levels of IL-4 induced the CP to produce CCL11, low levels of IL-4 induced BDNF, suggesting an unexpected dose-dependent opposite role of CP-associated IL-4 in mediating CNS homeostasis.

To further substantiate the dominance of IL-4 in the aged CP, we measured levels of arginase 1 ( $\arg 1)$, a known marker of epithelial Th2-mediated inflammation; in cases of asthma, chronic obstructive pulmonary disease, and cystic fibrosis, increased local arginase activity was shown to have detrimental effects on the airway epithelium (26). Moreover, chronic Th2-mediated inflammation of the airways induces remodeling of the epithelium and contributes to a progressive decline in lung function (27). We found $\arg 1 \mathrm{mRNA}$ and protein levels to be strongly up-regulated in the aged CP (Fig. $3 L$ and $M$ ) as well as in IL-4-treated CP cultures (Fig. $3 N$ ). Another effect of the exposure to IL-4 was dysregulation of the epithelial tight junctions (Fig. $3 N$ ), as observed here by staining for ZO-1, and as previously reported in the case of human lung epithelial cells (28). Histological examination of the $\mathrm{CP}$ revealed signs of hypertrophism in the aged $\mathrm{CP}$ epithelium (Fig. S3), a phenomenon described in the murine lung epithelium in response to IL-4 (29). Finally, we examined $\arg 1$ mRNA expression in the CP of young IFN- $\gamma \mathrm{R}-\mathrm{KO}$ animals and found it to be significantly up-regulated (Fig. S4). Together, these data indicate that the changes in the IL-4:IFN- $\gamma$ ratio in the CP of aged mice critically affect gene expression and morphology of the $\mathrm{BCSFB}$, and may potentially explain the age-related cognitive decline that was observed in correlation with elevated CCL11 levels in the blood and CSF (25).

Because brain aging and impaired hippocampal plasticity have been associated with elevation of parenchymal proinflammatory cytokines $(30,31)$ such as IL- $1 \beta$, IL- 6 , and TNF- $\alpha$, we assumed that the $\mathrm{CP}$ of aged mice is exposed to such a proinflammatory milleu. Examining the aged CP for the presence of proinflammatory cytokines revealed the elevated protein levels of IL- $1 \beta$ and IL-6 (Fig. S5), suggesting that the cytokine milieu of the aged parenchyma is signaling to the $\mathrm{CP}$, thereby possibly contributing to its dysfunction.

Lymphopenia-Induced Homeostasis-Driven Proliferation of T Cells Affects the CP and Hippocampus. The increased levels of IL-4 in the $\mathrm{CP}$ of aged mice without proper balance by IFN- $\gamma$ could reflect the well-characterized alternations in circulating immune cells during aging $(22,23)$. One way by which immunosenescence can be alleviated and memory T cells can be expanded is the induction of lymphopenia, which is followed by homeostasisdriven proliferation (HDP) of the residual T-cell population. We therefore induced HDP by irradiation of the peripheral organs while shielding the head. Following lymphopenia, we restored the hematopoietic cell lineage with syngeneic (pseudoautologous) bone marrow (BM) derived from genetically identical agematched donors. We expected that memory $\mathrm{T}$ cells that are present in the aged BM would provide, in addition to the residual T cells of the recipients, a source of memory cells for their expansion under these lymphopenic conditions. We first confirmed that aged BM contained memory $\mathrm{T}$ cells, and determined whether their specificity resembles that of cells found in $\mathrm{SCH}$ immunized spleen, old spleen, and naïve CP. We found that BM from aged mice contained significantly higher levels of memory $\mathrm{CD}^{+}{ }^{+}$cells relative to young animals (Fig. S6). Testing the clonotypic repertoire revealed that $\mathrm{T}$ cells in the $\mathrm{BM}$ were 
A
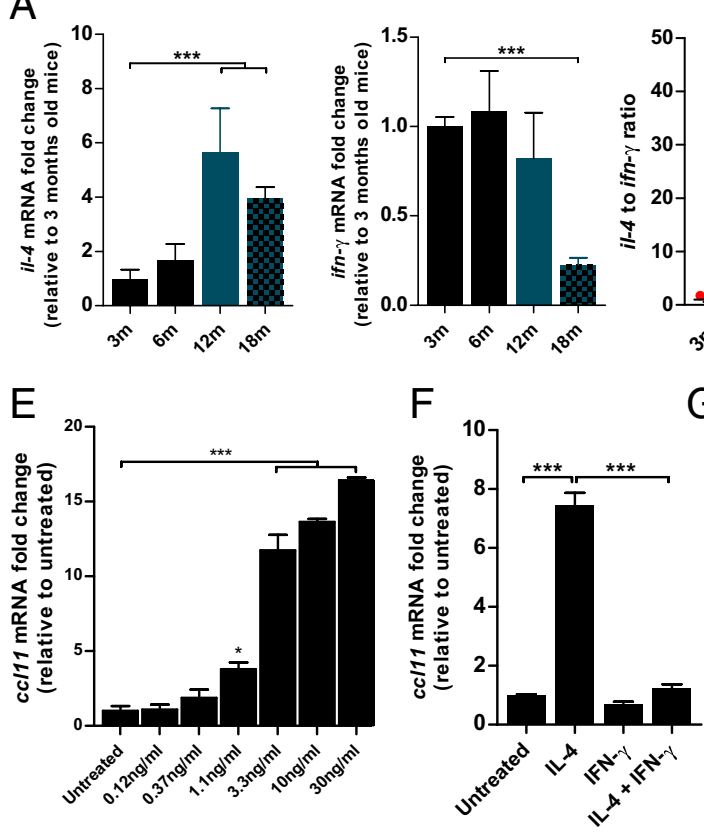

G

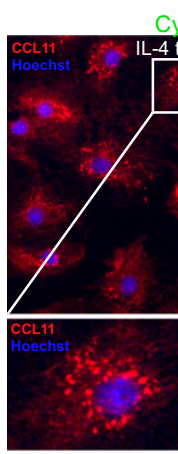

K

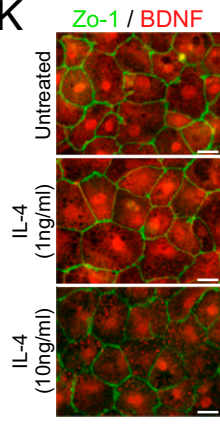

J

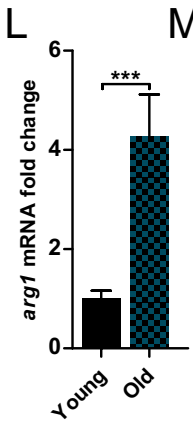

M
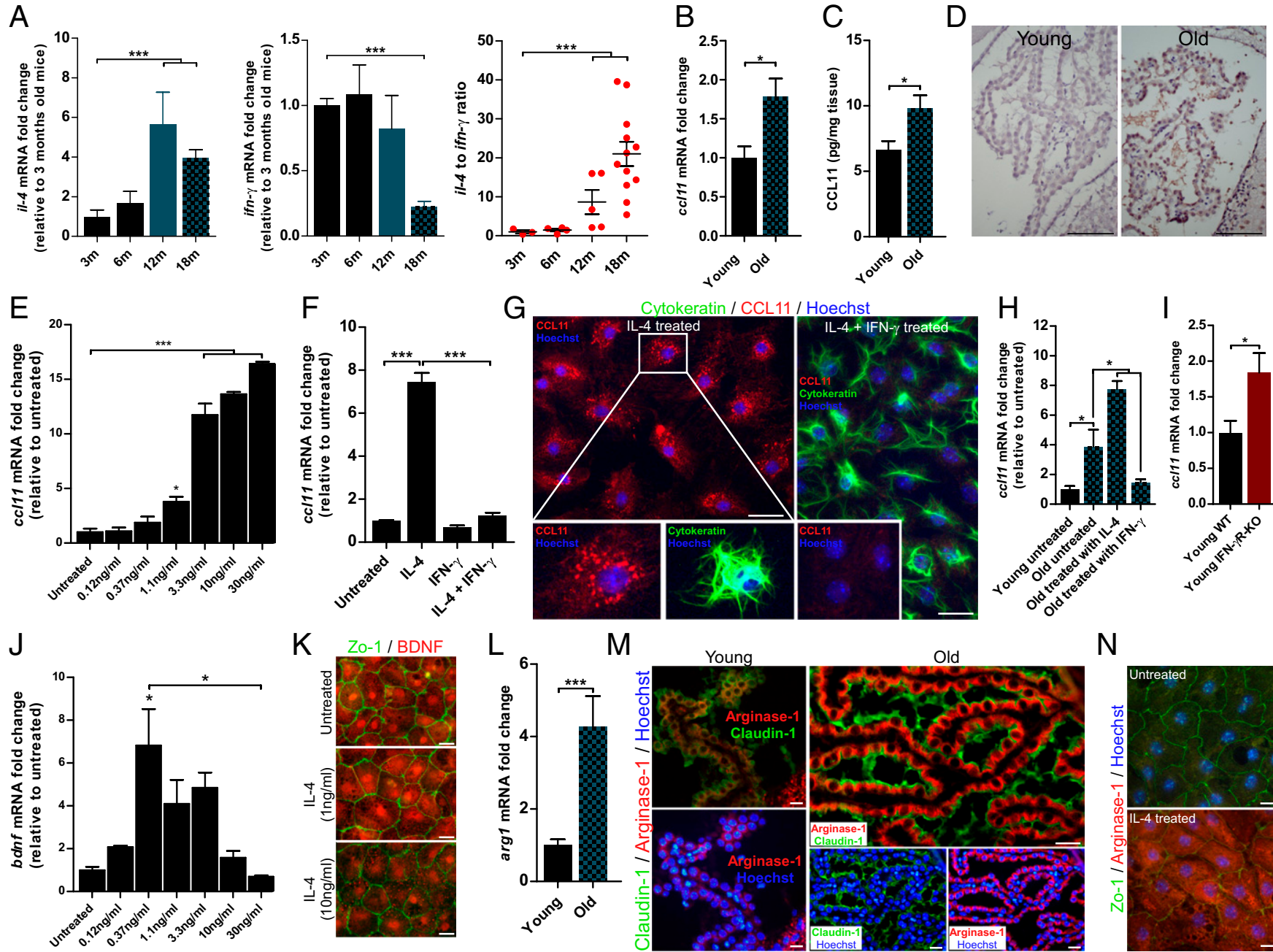
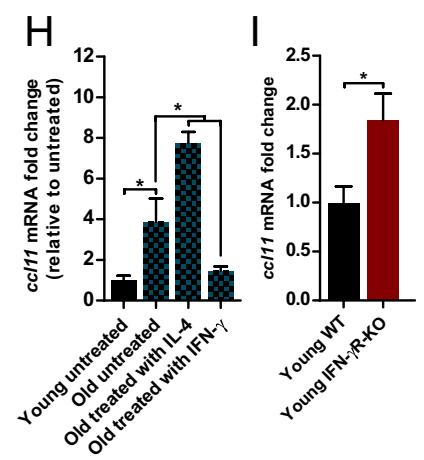

Fig. 3. Th2-mediated inflammation of the CP epithelium during the aging process. $(A)$ mRNA levels of $i l-4$, ifn- $\gamma$, and their ratio in the $C P$ of mice at the ages of 3, 6 , 12 , and $18 \mathrm{mo}$ ( $n=8-10$ per group; one-way ANOVA, Newman-Keuls post hoc). (B) ccl11 mRNA levels in the CP of young and old mice ( $n=5-6$ per group; Student's $t$ test). (C) CCL11 protein levels (pg/mg tissue) in the CP of young (3-mo-old) and old (20-to 22-mo-old) mice ( $n=16$ per group; Student's $t$ test). ( $D$ ) Representative micrographs showing immunohistochemical stainings for CCL11 in young and old mice. (Scale bars, $100 \mu \mathrm{m}$.) (E) ccl11 mRNA levels $24 \mathrm{~h}$ after the addition of IL-4 (untreated, and $0.12,0.37,1.1,3.3,10$, and $30 \mathrm{ng} / \mathrm{mL}$ ) of cultured CP epithelial cells ( $n=3$ per group; one-way ANOVA, Newman-Keuls post hoc; one representative experiment is shown out of three independently performed). $(F)$ ccl11 mRNA levels of cultured CP epithelial cells from young (3-mo-old) mice, $24 \mathrm{~h}$ after the addition of the cytokines IL-4 (10 ng/mL), IFN- $\gamma(100 \mathrm{ng} / \mathrm{mL})$, or their combination ( $n=3$ per group; one-way ANOVA, Newman-Keuls post hoc; one representative experiment is shown out of three independently performed). (G) Representative micrographs of cultured CP epithelial cells from young mice exposed to IL-4 (10 ng/mL) or the combination of IL-4 (10 ng/mL) and IFN- $\gamma(100 \mathrm{ng} / \mathrm{mL})$. Cells were treated with Brefeldin A $2 \mathrm{~h}$ before fixation and stained for the epithelial marker cytokeratin (green), CCL11 (red), and Hoechst nuclear staining (blue). (Scale bars, $25 \mu \mathrm{m}$.) $(H)$ ccl11 mRNA levels, measured by qPCR, of cultured CP epithelial cells from young and old mice, $24 \mathrm{~h}$ after the addition of the cytokines IL-4 $(10 \mathrm{ng} / \mathrm{mL})$ or IFN- $\gamma(100 \mathrm{ng} / \mathrm{mL})$ ( $n=3$ per group; one-way ANOVA, Newman-Keuls post hoc). (I) ccl11 mRNA levels, measured by qPCR, in the CP of young wild-type (3-mo-old) and age-matched IFN- $\gamma$ R-KO mice ( $n=5-6$ per group; Student's $t$ test). ( $)$ ) bdnf mRNA levels $24 \mathrm{~h}$ after the addition of IL-4 (untreated, and $0.12,0.37,1.1,3.3,10$, and $30 \mathrm{ng} / \mathrm{mL}$ ), measured by qPCR, of cultured CP epithelial cells ( $n=3$ per group; one-way ANOVA, Newman-Keuls post hoc; one representative experiment is shown out of three independently performed). (K) Representative micrographs of cultured CP epithelial cells from young (3-mo-old) mice, exposed to IL-4 at the concentrations of 1 or $10 \mathrm{ng} / \mathrm{mL}$ or untreated. Cells were stained for ZO-1 (green) and for BDNF (red). (Scale bars, $25 \mu \mathrm{m}$.) ( $L$ ) arg1 mRNA levels, measured by qPCR, in the CP of young (3-mo-old) and old (22-mo-old) mice ( $n=5-6$ per group; Student's $t$ test). ( $M$ ) Representative micrographs showing staining for Claudin 1 (tight junctions; green), arginase 1 (red), and Hoechst nuclear staining (blue). (Scale bars, $25 \mu \mathrm{m}$.) ( $N$ ) Representative micrographs of cultured CP epithelial cells from young (3-mo-old) mice exposed to IL-4 or left untreated. Cells were stained for ZO-1 (green), arginase 1 (red), and Hoechst nuclear staining (blue). (Scale bars, $25 \mu \mathrm{m}$.) Bar graphs throughout the figure show mean $\pm \mathrm{SE}$ of each group $(\star P<0.05 ; \star \star P<0.01 ; * \star \star P<0.001)$.

significantly enriched for CNS specificity (Fig. $4 A$ ). To verifiy that following $\mathrm{BM}$ transplantation, the $\mathrm{T}$ cells from the aged donor BM repopulated the recipient lymphoid organs, donor BM cells were labeled with the intracellular fluorescent dye carboxyfluorescein succinimidyl ester (CFSE) before transplantation. $\mathrm{BM}$-derived $\mathrm{T}$ cells migrated to the spleen, where they proliferated over time (Fig. S7); in young and aged recipients, these $\mathrm{T}$ cells could be detected in the spleen and cervical LNs, and showed dilution of the CFSE label (indicating proliferation).

In previous experiments, using a similar protocol of HDP and $\mathrm{BM}$ transplantation from aged donors, we demonstrated cognitive improvement in aged animals (32). Because our protocol involved aged $\mathrm{BM}$ as our source of additional memory $\mathrm{T}$ cells beyond the irradiation remnants that undergo proliferation following irradiation-induced lymphopenia, we verified here the cognitive improvement previously reported is indeed attributable to the transferred T cells that reside in the donor BM. To this end, HDP was induced in cognitively impaired aged mice that were either transplanted with aged-matched BM or with the same BM depleted of T cells. The mice were tested again, $8 \mathrm{wk}$ later, in the Morris water maze (MWM) for their hippocampus-dependent spatial learning/memory relative to young mice. Old mice 

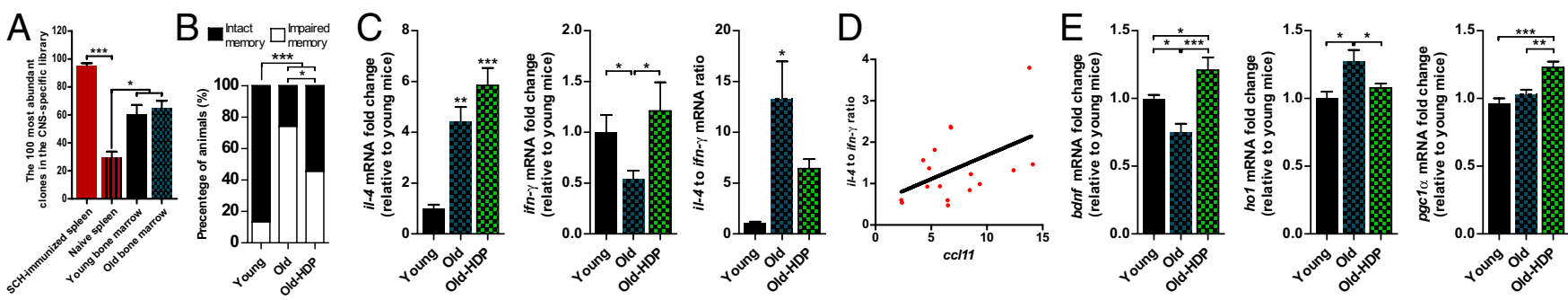

Fig. 4. Syngeneic homeostasis-driven proliferation effects on the CP and hippocampus. $(A)$ The 100 most abundant TCR $\beta$ clones from the spleen and bone marrow of young (3-mo-old) and old (22-mo-old) mice were examined for their presence in the CNS-specific clonal library ( $n=8$ per group; one-way ANOVA, Newman-Keuls post hoc). (B) Morris water maze test for young mice (3-mo-old), untreated old (22-mo-old), and aged littermates (old-HDP) 3 months following homeostasis-driven proliferation. Mice were divided into intact or impaired memory groups according to their MWM score, where an average latency time of above $60 \mathrm{~s}$ on the fourth day of acquisition was classified as "impaired memory" and a latency of less than 60 s was defined as "intact memory" ( $n=10-14$ per group; $\chi^{2}$ test). ( $C$ ) $i l-4$ and ifn- $\gamma$ mRNA levels and ratio in the CP of young, old, and old-HDP mice (one-way ANOVA, Newman-Keuls post hoc). (D) Correlation analysis between the CP mRNA levels of il-4:ifn- $\gamma$ ratio and ccl11 $\left(n=17\right.$; Pearson $\left.r^{2}=0.2294\right)$. $(E) b d n f$, ho1, and pgc1 $\alpha$ mRNA levels in the hippocampus of young, old, and old-HDP mice (one-way ANOVA, Newman-Keuls post hoc). Bar graphs throughout the figure show mean \pm SE of each group $(* P<0.05 ; * * P<0.01 ; * * \star P<0.001)$.

receiving $\mathrm{BM}$ depleted of $\mathrm{T}$ cells failed to reestablish learning/ memory skills (Fig. S8).

Based on the above results, we adopted here the same HDP procedure using pseudoautologous aged BM to examine whether such peripheral immunomodulation, which leads to cognitive improvement, would be associated with changes of the IL-4:IFN- $\gamma$ ratio at the $\mathrm{CP}$. Before excising the $\mathrm{CP}$ and the hippocampi of the animals, we verified using MWM that the treatment was indeed beneficial for learning/memory 3 mo following transplantation. The proportion of aged animals with impaired spatial learning/ memory was $74 \%$, as commonly found (33); among the aged animals subjected to HDP, the proportion of cognitively impaired animals was reduced to $45 \%$ (Fig. $4 B$ ). Testing the $\mathrm{CP}$ of the same mice for IL-4 and IFN- $\gamma$ expression showed that in the old animals following HDP, the IL-4:IFN- $\gamma$ ratio was similar to that found in young animals (Fig. $4 C$ ). The causal connection between the IL-4:IFN- $\gamma$ ratio and CCL11 levels, and thus the impact on cognitive ability, was further demonstrated by the positive correlation found between the IL-4:IFN- $\gamma$ ratio and CCL11 levels in the CP of old animals (treated and untreated) (Fig. 4D).

Because spatial learning/memory is hippocampus-dependent, we also tested whether the HDP-induced changes in the CP cytokine balance were also reflected in the expression of plasticityrelated molecules in the hippocampi of these mice. We found that $b d n f$ levels, repeatedly linked with age-associated loss of cognitive function (34), were down-regulated with age and restored following HDP (Fig. 4E). In addition, we measured the expression of heme oxygenase 1 (hol), previously linked to aging of the hippocampus (35), and found it to be up-regulated in the aged group compared with the young animals. HDP reduced hol in aged animals to its expression levels in young animals (Fig. $4 E$ ). We also examined whether rejuvenation of the $\mathrm{CP}$ cytokine balance was associated with elevation of PPAR $\gamma$ coactivator $1 \alpha(p g c 1 \alpha)$, a key regulator in coping with reactive oxygen species and a hippocampal neuroprotective mediator (36). Old animals after HDP had significantly higher levels of hippocampal pgcl $\alpha$ (Fig. 4E), possibly correlating with their improved cognitive ability. Taken together, this peripheral immunomodulation, which improved cognitive ability and affected cytokine balance at the CP, also correlated with molecular changes at the hippocampus.

\section{Discussion}

Here we show that the choroid plexus epithelium, an active interface between blood circulation and the brain, is populated by CNS-specific effector memory-type $\mathrm{CD} 4^{+} \mathrm{T}$ cells. We further demonstrate that with aging, the overall specificity of $\mathrm{CP}$ resident $\mathrm{T}$ cells toward CNS antigens was maintained, although the cytokine-chemokine balance changed toward a Th2-like epithelial inflammation, with detrimental consequences to brain function.

CNS specificity plays a part in the beneficial effector function of $\mathrm{T}$ cells in CNS plasticity and repair $(2-4,6,37,38)$. Experimentally, $\mathrm{T}$ cells involvement in brain plasticity was found to be dependent on their CNS-specificity (2). Here, we found that the CP, under physiological conditions, retains a broad repertoire of CNS-specific $\mathrm{CD} 4^{+} \mathrm{T}$-cell clones. These $\mathrm{T}$ cells, upon recognition and reactivation by their cognate antigens, potentially presented to them by the Flt ${ }^{+}$dendritic cells found in the CP (39), either enter the CSF or remain in the CP $(40,41)$. Although TCR-seq of the CP allowed us to identify CNS-specific clonotypic enrichment both in young and aged mice, other parameters of age-related changes of the TCR repertoire should not be ruled out. In addition, the appearance of such clones in other organs, such as the lung (42), should be further investigated.

One of the most prominent features of aging is a shift toward a dominance of the helper Th2-related response. Accordingly, murine models of aging were shown to manifest age-associated dysregulation in cytokine production, specifically involving reduced production of IFN- $\gamma$, and increased production of IL- 4 $(23,33)$. Examining the local effector cytokine balance in the $\mathrm{CP}$ revealed a gradual increase in IL-4:IFN- $\gamma$ ratio during aging, which may reflect the general condition of the aged immune system, further supporting the contention that the immune response within the $\mathrm{CP}$ is an integral part of systemic immunity. Notably, the ongoing T-cell dialogue in this compartment was also manifested by the $\mathrm{CD}^{+}$clonotypic enrichment of CNS-specific TCR in the CP of mice immunized with CNS antigens, but not of mice immunized with ovalbumin.

The age-related change in $\mathrm{CP}$ cytokine milieu was found here to critically affect this compartment. When not properly balanced by IFN- $\gamma$, high levels of IL-4 induced the CP epithelium to produce CCL11, a chemokine associated with age-related cognitive impairments that accumulates at the aged CSF (25) and can directly interfere with IL-4 signaling (43). Because cognitive tasks lead to accumulation of IL-4-producing T cells in the meningeal spaces of the brain (8) and IL-4 has a beneficial role in learning and memory, our findings highlight a dual role of IL-4 in the CNS, that may depend on its spatial localization and dosing. Accordingly, epithelial IL-4-induced CCL11 might impair cognition by its potential ability to suppress IL-4 originated from the meninges, as recently suggested (44). Importantly, however, it is very likely that not only meningeal IL-4 supports brain plasticity, but also basal/low levels of IL-4 at the CP are part of a lifelong role of this compartment in keeping CNS homeostasis, as was manifested here by BDNF expression by $\mathrm{CP}$ epithelial cells. If this is the case, the age-related overwhelming levels of IL-4 at the CP have a negative outcome for the functioning brain, not only because of induction of CCL11 but also because of dysregulation of BDNF and possibly other $\mathrm{CP}$ derived neurotrophic factors. Notably, the CP produces BDNF following CNS trauma (45) and chronic stress (46).

The fact that CCL11 was found here to be produced by the CP epithelium, and specifically in response to high levels of IL-4, coupled with the negative effects of CCL11 on brain functional plasticity, supports our hypothesis that the loss of cognitive ability in old mice is a reflection of dysregulation of epithelium-T 
cell cross-talk at the CP. Such Th2-like inflammation is known in epithelial pathologies outside the CNS, such as the lung epithelium in asthma (47). Intriguingly, examining the aged CP epithelium in analogy to the asthmatic Th2-inflamed lung epithelium revealed similarities in mechanistic and functional dysregulation that may reflect common disease-like processes amenable to immunomodulation.

Previous studies carried out by our group have demonstrated that $\mathrm{T}$ cell-mediated immunity supports hippocampus-dependent cognitive ability, and that age-related memory loss may be reversed, at least to some extent, by peripheral immunomodulation in the form of lethal irradiation followed by syngeneic bone marrow transplantation $(2,34)$. Using the same paradigm here, we demonstrate that this peripheral immunomodulation could locally influence the CP, alleviating age-related overwhelming IL-4 levels, and suggest a mechanism as to how these changes affect cognitive ability.

In summary, the present study identifies the choroid plexus and its CNS-specific immunity as unique players that functionally link brain aging and immunosenescence. We further show that modulating the cytokine imbalance in this compartment has the potential to partially restore memory function. It is therefore possible that targeting treatment for brain aging, based on immunomodulating

1. Wolf SA, et al. (2009) CD4-positive T lymphocytes provide a neuroimmunolog ical link in the control of adult hippocampal neurogenesis. J Immunol 182(7): 3979-3984.

2. Ziv $Y$, et al. (2006) Immune cells contribute to the maintenance of neurogenesis and spatial learning abilities in adulthood. Nat Neurosci 9(2):268-275.

3. Kipnis J, Cohen H, Cardon M, Ziv Y, Schwartz M (2004) T cell deficiency leads to cognitive dysfunction: Implications for therapeutic vaccination for schizophrenia and other psychiatric conditions. Proc Natl Acad Sci USA 101(21):8180-8185.

4. Moalem G, et al. (1999) Autoimmune $T$ cells protect neurons from secondary degeneration after central nervous system axotomy. Nat Med 5(1):49-55.

5. Olsson T, Lidman O, Piehl F (2003) Harm or heal-Divergent effects of autoimmune neuroinflammation? Trends Immunol 24(1):5-6, author reply 7-8.

6. Hofstetter $\mathrm{HH}$, et al. (2003) Autoreactive T cells promote post-traumatic healing in the central nervous system. J Neuroimmunol 134(1-2):25-34.

7. Engelhardt B, Ransohoff RM (2005) The ins and outs of T-lymphocyte trafficking to the CNS: Anatomical sites and molecular mechanisms. Trends Immuno/ 26(9):485-495.

8. Derecki NC, et al. (2010) Regulation of learning and memory by meningeal immunity: A key role for IL-4. J Exp Med 207(5):1067-1080.

9. Lewitus GM, Cohen H, Schwartz M (2008) Reducing post-traumatic anxiety by im munization. Brain Behav Immun 22(7):1108-1114.

10. Clarke RM, et al. (2008) A pivotal role for interleukin-4 in atorvastatin-associated neuroprotection in rat brain. J Biol Chem 283(4):1808-1817.

11. Lyons A, Griffin RJ, Costelloe CE, Clarke RM, Lynch MA (2007) IL-4 attenuates the neuroinflammation induced by amyloid-beta in vivo and in vitro. J Neurochem 101(3): 771-781.

12. Cao C, et al. (2009) Abeta-specific Th2 cells provide cognitive and pathological benefits to Alzheimer's mice without infiltrating the CNS. Neurobiol Dis 34(1):63-70.

13. Butovsky O, et al. (2006) Microglia activated by IL-4 or IFN-gamma differentially induce neurogenesis and oligodendrogenesis from adult stem/progenitor cells. Mol Cell Neurosci 31(1):149-160.

14. Emerich DF, Skinner SJ, Borlongan CV, Vasconcellos AV, Thanos CG (2005) The choroid plexus in the rise, fall and repair of the brain. BioEssays 27(3):262-274.

15. Sallusto F, Geginat J, Lanzavecchia A (2004) Central memory and effector memory T cel subsets: Function, generation, and maintenance. Annu Rev Immunol 22:745-763.

16. de Graaf MT, et al. (2011) Central memory $C D 4^{+}$T cells dominate the normal cerebrospinal fluid. Cytometry B Clin Cytom 80(1):43-50.

17. Kivisäkk $P$, et al. (2003) Human cerebrospinal fluid central memory CD4 ${ }^{+} T$ cells: Ev idence for trafficking through choroid plexus and meninges via P-selectin. Proc Nat Acad Sci USA 100(14):8389-8394.

18. Freeman JD, Warren RL, Webb JR, Nelson BH, Holt RA (2009) Profiling the T-cell receptor beta-chain repertoire by massively parallel sequencing. Genome Res 19(10) 1817-1824.

19. Robins HS, et al. (2009) Comprehensive assessment of T-cell receptor beta-chain diversity in alphabeta T cells. Blood 114(19):4099-4107.

20. Ndifon $W$, et al. (2012) Chromatin conformation governs T-cell receptor J $\beta$ gene segment usage. Proc Natl Acad Sci USA 109(39):15865-15870.

21. Linton PJ, Dorshkind K (2004) Age-related changes in lymphocyte development and function. Nat Immuno/ 5(2):133-139.

22. Alberti $S$, et al. (2006) Age-dependent modifications of type 1 and type 2 cytokines within virgin and memory CD4 ${ }^{+}$T cells in humans. Mech Ageing Dev 127(6):560-566.

23. Shearer GM (1997) Th1/Th2 changes in aging. Mech Ageing Dev 94(1-3):1-5.

24. Bloemen K, et al. (2007) The allergic cascade: Review of the most important molecules in the asthmatic lung. Immunol Lett 113(1):6-18. the BCSFB, may lead to hitherto unexplored approaches to reverse or arrest dementia, other age-related cognitive deficits, or neurodegenerative diseases.

\section{Materials and Methods}

Young and aged C57BL/6 and IFN- $\gamma$ R1-KO mice were handled according to regulations formulated by The Weizmann Institute's Animal Care and Use Committee and maintained in a pathogen-free environment. A detailed description of all experimental procedures, including flow cytometry, active immunizations, TCR-Seq, primary culture of choroid plexus, immunohistochemistry, immunocytochemistry, multiplex cytokine analysis, RNA and cDNA preparation for quantitative PCR, HDP procedure, cognitive tests, and statistical analysis can be found in SI Materials and Methods.

ACKNOWLEDGMENTS. We thank Dr. Gilad Kunis for technical assistance, Dr. Shelley Schwarzbaum for editing the manuscript, Dr. Hillary Voet for statistical consultation, and Margalit Azoulay for animal handling. This research was supported by a European Research Council Grant Award, a Seventh Framework Programme HEALTH-2011 Grant (to M.S.), and by the Intramural Research Program of the National Institute on Aging (to M.P.M.). N.F. is the incumbent Pauline Recanati Career Development Chair of Immunology. M.S. holds The Maurice and Ilse Katz Professorial Chair in Neuroimmunology.

25. Villeda SA, et al. (2011) The ageing systemic milieu negatively regulates neurogenesis and cognitive function. Nature 477(7362):90-94.

26. Maarsingh H, Pera T, Meurs H (2008) Arginase and pulmonary diseases. Naunyn Schmiedebergs Arch Pharmacol 378(2):171-184.

27. Postma DS, Timens W (2006) Remodeling in asthma and chronic obstructive pulmonary disease. Proc Am Thorac Soc 3(5):434-439.

28. Ahdieh M, Vandenbos T, Youakim A (2001) Lung epithelial barrier function and wound healing are decreased by IL-4 and IL-13 and enhanced by IFN-gamma. Am J Physiol Cell Physiol 281(6):C2029-C2038.

29. Rankin JA, et al. (1996) Phenotypic and physiologic characterization of transgenic mice expressing interleukin 4 in the lung: Lymphocytic and eosinophilic inflammation without airway hyperreactivity. Proc Natl Acad Sci USA 93(15):7821-7825.

30. Dantzer R, O'Connor JC, Freund GG, Johnson RW, Kelley KW (2008) From inflammation to sickness and depression: When the immune system subjugates the brain. Nat Rev Neurosci 9(1):46-56.

31. Ekdahl CT, Claasen JH, Bonde $\mathrm{S}$, Kokaia Z, Lindvall O (2003) Inflammation is detrimental for neurogenesis in adult brain. Proc Natl Acad Sci USA 100(23):13632-13637.

32. Ron-Harel N, et al. (2008) Age-dependent spatial memory loss can be partially restored by immune activation. Rejuvenation Res 11(5):903-913.

33. Whalley L, Deary IJ, Appleton CL, Starr JM (2004) Cognitive reserve and the neurobiology of cognitive aging. Ageing Res Rev 3(4):369-382.

34. Kesslak JP, So V, Choi J, Cotman CW, Gomez-Pinilla F (1998) Learning upregulates brain-derived neurotrophic factor messenger ribonucleic acid: A mechanism to facilitate encoding and circuit maintenance? Behav Neurosci 112(4):1012-1019.

35. Nicolle MM, et al. (2001) Signatures of hippocampal oxidative stress in aged spatial learning-impaired rodents. Neuroscience 107(3):415-431.

36. St-Pierre J, et al. (2006) Suppression of reactive oxygen species and neurodegeneration by the PGC-1 transcriptional coactivators. Cell 127(2):397-408.

37. Kipnis J, Gadani S, Derecki NC (2012) Pro-cognitive properties of T cells. Nat Rev Immunol 12(9):663-669.

38. Schwartz M, Shechter R (2010) Protective autoimmunity functions by intracranial immunosurveillance to support the mind: The missing link between health and disease. Mol Psychiatry 15(4):342-354.

39. Anandasabapathy $\mathrm{N}$, et al. (2011) Flt3L controls the development of radiosensitive dendritic cells in the meninges and choroid plexus of the steady-state mouse brain. J Exp Med 208(8):1695-1705.

40. Bartholomäus I, et al. (2009) Effector T cell interactions with meningeal vascular structures in nascent autoimmune CNS lesions. Nature 462(7269):94-98.

41. McMenamin PG (1999) Distribution and phenotype of dendritic cells and resident tissue macrophages in the dura mater, leptomeninges, and choroid plexus of the rat brain as demonstrated in wholemount preparations. J Comp Neurol 405(4): 553-562.

42. Odoardi F, et al. (2012) T cells become licensed in the lung to enter the central nervous system. Nature 488(7413):675-679.

43. Stevenson NJ, et al. (2009) CCL11 blocks IL-4 and GM-CSF signaling in hematopoietic cells and hinders dendritic cell differentiation via suppressor of cytokine signaling expression. J Leukoc Biol 85(2):289-297.

44. Ransohoff RM (2011) Ageing: Blood ties. Nature 477(7362):41-42.

45. Borlongan CV, et al. (2004) CNS grafts of rat choroid plexus protect against cerebral ischemia in adult rats. Neuroreport 15(10):1543-1547.

46. Sathyanesan M, et al. (2012) A molecular characterization of the choroid plexus and stress-induced gene regulation. Transl Psychiatry 2:e139.

47. Cockcroft DW, Davis BE (2006) Mechanisms of airway hyperresponsiveness. J Allergy Clin Immunol 118(3):551-559, quiz 560-561. 